\title{
Obesity, Waist Circumference, Weight Change, and the Risk of Psoriasis in US Women
}

\author{
Sandeep Kumar, MD², Jiali Han, PhD ${ }^{1,2,4}$, Tricia Li, MD, MS ${ }^{1}$, Gary Curhan, MD, ScD ${ }^{1,4}$, \\ Hyon K. Choi, MD, DrPH ${ }^{1,3}$, and Abrar A. Qureshi, MD, MPH ${ }^{1,2}$ \\ ${ }^{1}$ Channing Laboratory, Department of Medicine, Brigham and Women's Hospital and Harvard \\ Medical School, Boston, MA \\ 2Department of Dermatology, Brigham and Women's Hospital and Harvard Medical School, \\ Boston, MA \\ ${ }^{3}$ Department of Rheumatology, Boston University, Boston, MA \\ ${ }^{4}$ Department of Epidemiology, Harvard School of Public Health, Boston, MA
}

\begin{abstract}
Background-Psoriasis is a common chronic inflammatory skin disorder. Higher adiposity may increase the risk of psoriasis, but prospective data on this association are scarce. One prospective study showed that increased adiposity increased the risk of incident psoriasis in younger women, but no data are available in older women.
\end{abstract}

Methods-We prospectively examined the associations between body mass index (BMI), weight change, waist and hip circumference and risk of incident psoriasis in 67,300 women over a 12year period (1996-2008) in the Nurses' Health Study. The primary outcome was self-reported, physician-diagnosed psoriasis.

Results-During the 12 years of follow-up, there were a total of 809 incident psoriasis cases. There was a graded positive association between BMI (both baseline and updated) and the risk of psoriasis (both $\mathrm{P}$ values for trend $<0.0001$ ). Compared to women with updated BMI of $<25$, the multivariate RRs (relative risks) of incident psoriasis were 1.21 (95\% CI, 1.03-1.43) for a BMI of 25.0 to $29.9,1.63$ (95\% CI, 1.33-2.00) for a BMI of 30.0 to 34.9, and 2.03 (95\% CI, 1.58-2.61) for a BMI of 35.0 or greater. Higher waist circumference, hip circumference, and waist-hip ratio were associated with a higher risk of incident psoriasis, but became non-significant after additionally adjusting for BMI. The BMI at age of 18 years was not associated with the risk of psoriasis.

Corresponding Author: Abrar A. Qureshi, MD, MPH, Assistant Professor, Harvard Medical School, Department of Dermatology, Brigham and Women's Hospital, 221 Longwood Avenue, Boston, MA 02115, T 617 525-5584, F 617 525-5571, abrar.qureshi@channing.harvard.edu.

Author Contributions: Dr Qureshi had full access to all the data in the study and takes responsibility for the integrity of the data and the accuracy of the data analysis. Study concept and design: JH, AQ. Acquisition of data: JH, AQ. Analysis and interpretation of data: JH, TL. Drafting of the manuscript: SK, JH. Critical revision of the manuscript for important intellectual content: SK, JH, GC, HC, AQ. Statistical analysis: JH, TL. Obtained funding: JH, AQ. Administrative, technical, or material support: JH, AQ. Study supervision: JH, AQ.

Financial disclosure: Relationships relevant to this manuscript: None. All other relationships: Dr. Qureshi has served as a consultant to Amgen and Genentech. Drs. Kumar, Choi, Han, Li and Curhan have no conflicts of interest to declare. 
Weight gain since the age of 18 years was associated with an increased risk of psoriasis and RR of $10 \mathrm{lb}$ gain was 1.08 (95\% CI, 1.06-1.11; $\mathrm{p}<0.0001)$.

Conclusion-This large prospective study indicates that higher BMI and weight gain are risk factors for incident psoriasis in older US women.

\section{INTRODUCTION}

Psoriasis is a common chronic debilitating systemic inflammatory disorder that affects millions of individuals worldwide. The prevalence of psoriasis was noted to be 2.5 percent in Caucasians and 1.3 percent in African-Americans in a population-based study in the US [1]. A common comorbidity associated with psoriasis is psoriatic arthritis, an inflammatory arthritic condition seen in $25 \%$ to $34 \%$ of patients with psoriasis [2]. Many more comorbidities have been described in association with psoriasis $[3,4,5]$. The exact etiology remains unknown, but both genetic and environmental factors have been implicated in the onset and progression of this chronic immune-mediated disease.

Regardless of etiology, obesity confers a mortality risk, with life expectancy inversely related to an individual's degree of adiposity, and independent of other negative health influences such as smoking [6]. Obesity is a medical condition in which excess body fat has accumulated to the extent that it may have an adverse health effects, such as decreased longevity, diabetes mellitus, orthopedic and respiratory disease and neoplasia $[7,8]$. Of note, $31 \%$ of all adults in the U.S.A. are classified as obese [9]. Multiple cross-sectional studies have demonstrated the association between psoriasis and obesity $[10,11,12,13]$.

Previous cross-sectional studies, case-control studies and the other prospective study from our group, have all consistently suggested a significant link between increased adiposity and psoriasis. In 1986, an inpatient Swedish study reported that women with psoriasis had a higher prevalence of obesity $(P<0.001)$ [14]. A correlation between psoriasis and obesity was observed in 1995 by Henseler and Christophers [15]. Later, large clinical trials evaluating biological agents for moderate to severe psoriasis reported that subjects were much heavier than the population norm [16,17]. In 2005, Naldi et al demonstrated that the risk of psoriasis was directly related to high BMI in a case-control study involving 560 patients with newly diagnosed psoriasis and 690 controls [11]. The prevalence of psoriasis was approximately twice as high in individuals with a BMI of 30 or greater compared with a BMI of less than 26 (multivariate odds ratio, 1.9; 95\% CI, 1.2-2.8) [11]. Another study, the Utah Psoriasis Initiative study, suggested that in individual patients obesity usually follows psoriasis, implying that psoriatic inflammation contributes to the obese state. The prevalence of obesity in the patients with psoriasis enrolled in the study was nearly twice as high as that in the general population ( $34 \%$ vs $18 \% ; P<0.001)$ [10].Confirming these analyses, a more broadly representative cross-sectional study in the U.K. underscored that patients with psoriasis were more likely to be obese, and that obesity was more prevalent in patients with severe psoriasis than mild psoriasis [6]. Huerta et al in 2007 reportedthat overweight individuals had slightly increased risk of developing psoriasis in a cohort study with nested case-control analysis which showed that adjusted OR (95\% CI) was 1.33 (1.16-1.52) in subjects with BMI >-30 in comparison to 1.11 (1.00-1.24) in subjects with BMI 25-29 [12]. 
The only prospective study published indicated that increased adiposity and weight gain are strong risk factors for incident psoriasis in younger women, but we had no data on incident psoriasis in older women [18]. To address these issues, we prospectively evaluated the association between BMI, weight change, waist circumference, hip circumference, waist-hip ratio, and incident psoriasis in a cohort of US women.

\section{MATERIAL AND METHODS}

\section{Study population}

The Nurses' Health Study (NHS) was established in 1976, when 121,700 female U.S. registered nurses between the ages of 30 and 55 residing in 11 larger U.S. states completed and returned the initial self-administered questionnaire on their medical histories and baseline health related exposures, forming the basis for the NHS cohort. These states represent the Northeast (Massachusetts, Connecticut, New York, New Jersey, Pennsylvania, Maryland), the North-central (Ohio, Michigan), the West (California), and the South (Texas, Florida). The population is predominantly white, reflecting the ethnic background of women entering nursing in the U.S. in the 1950's and 1960's. Biennial questionnaires with collection of exposure information on risk factors and nutritional assessments (every 4 years since 1980) have been collected prospectively. Along with exposures assessed every 2 years, outcome data with appropriate follow-up of reported disease events are collected. After more than 30 years, approximately $90 \%$ of participants continue to complete questionnaires.

\section{Ascertainment of covariates}

Information on weight and height was obtained from the 1976 questionnaire. Participants then reported their current weight on the biennial mailed questionnaires. Information on weight at the age of 18 years was obtained from the 1980 questionnaire.

Body mass index (BMI, calculated as weight in kilograms divided by height in meters squared) was analyzed at multiple time points (age of 18 years, baseline, and updated every 2 years). Waist and hip circumference reported in 1996 was used in the analysis. Weight change since the age of 18 years was calculated by subtracting the current weight (updated biennially) from the weight at age 18 . The accuracy of self-reported anthropometric measures was evaluated among 140 NHS participants by having trained technicians visit those participants twice [19]. After adjustment for age and within-person variability, the Pearson correlation coefficient between self-report and the average of the 2 technician measurements was 0.98 for weight, 0.91 for waist circumference, and 0.87 for hip circumference [19].

The baseline and biennial follow-up questionnaires asked about smoking status and alcohol intake. The reproducibility and validity of this questionnaire for alcohol intake have been previously documented in the NHS [5,20]. Information on physical activity was also obtained from the cohort questionnaires. Physical activity measures were described in detail previously [21]. We used the information obtained in the 1996 questionnaire and categorized into quintiles in the analysis. 


\section{Ascertainment of outcomes (psoriasis)}

In the 2008 questionnaire, we asked the question of "have you ever had any of these clinician-diagnosed illnesses or procedures?" The disease list included psoriasis. We asked the first diagnosis in different year categories: 1997 or before, 1998-2001, 2002-2005, 2006-2007, or 2008+.

The validity of self-reported diagnosis of psoriasis is high in such a medically sophisticated population. The validation rate of psoriasis in the Nurses' Health Study II (a similar cohort) is $92 \%$ for psoriasis. (Manuscript under submission).

\section{Statistical analyses}

Participants who did not answer the questions on the diagnosis of psoriasis were excluded. We computed person-time of follow-up for each participant from the return date of the 1996 questionnaire. Psoriasis cases with diagnosis after 1998 were included in the analysis. Accumulation of follow-up time ceased at the first report of psoriasis, death from another cause, or the return of 2008 questionnaire, whichever came first. We used updated BMI prior to the year of the diagnosis of psoriasis.

We used Cox proportional hazards models to calculate the relative risk (RR) and 95\% confidence intervals (CIs). We classified BMI into 5 categories (<18.5, 18.5-24.9, 25-29.9, 30-34.9 and 35+). The World Health Organization classifies the last 3 categories as overweight, obesity class I, and obesity class II, respectively [20]. We limited our presentation for $18.5+$ groups due to a very small number of cases in the $<18.5$ group. Weight change was analyzed in 5 categories (>-5 lb, -5.0 to $4.9 \mathrm{lb}, 5.0$ to $19.9 \mathrm{lb}, 20.0$ to $34.9 \mathrm{lb}$, and $35 \mathrm{lb}+)$. We categorized waist circumference, hip circumference and waist-hip ratios into tertiles. Multivariate models were adjusted for age (continuous), smoking status (never, current, or past smokers), and alcohol intake (6 categories: none, 1-4 g/d, 5-9 g/d, $10-14 \mathrm{~g} / \mathrm{d}, 15-29 \mathrm{~g} / \mathrm{d}$, and >_30 g/d). The BMI analysis additionally controlled for physical activity (quintiles). The analysis of weight change from the age of 18 years was also adjusted for BMI at age 18. The analysis of waist circumference, hip circumference, and waist-hip ratio were also additionally adjusted for continuous BMI. We constructed interaction terms between the risk factors and calendar year and used likelihood ratio tests to assess the significance of these interaction terms, which suggested that the assumptions of proportional hazards were met.

The Partners Health Care System (Boston, Massachusetts) institutional review board approved this study. Return of a completed questionnaire was accepted by the institutional review board as implied informed consent.

\section{RESULTS}

\section{BASELINE CHARACTERISTICS}

We studied a total of 67,300 women for a combined follow- up time of 886,321 personyears, during which there were a total 809 newly diagnosed cases of psoriasis. The baseline characteristics of the cohort in 1996 according to BMI are shown in Table 1. As expected, 
BMI at 18, weight gain since age 18, waist and hip circumference, and waist-hip ratio varied in the same direction. With increasing category of BMI at baseline, alcohol intake and cigarette smoking tended to decrease.

\section{BMI AND PSORIASIS}

We found a graded association between baseline BMI measured in 1996 and the risk of psoriasis (Table 2). When we compared baseline BMI of 18.5-24.9 (reference group), the multivariate RR of incidence of psoriasis for a BMI of 35.0 or greater was 1.63 (95\% CI, 1.24-2.14). When we analyzed BMI updated every 2 years, compared to women with a BMI of 18.5 to 24.9 , the multivariate RR of incident psoriasis was 2.03 (95\% CI, 1.58-2.61) for a BMI of 35.0 or greater.

The multivariate RR with 1 BMI unit increment was 1.04 (p for 1 unit increment, <0.0001) in both the above analysis of BMI and psoriasis. There was no material difference in ageadjusted RRs and multivariate RRs adjusted for age, alcohol consumption, smoking status, waist-hip ratio, and physical activity. Additional adjustment for BMI at age of 18 years did not change the results.

In addition, we did not observe a strong association between BMI at age of 18 years and risk of psoriasis. The multivariate RR with 1 BMI unit increment was 1.02 (95\% CI, 0.99-1.04) $(\mathrm{P}, 0.17)$.

\section{WAIST CIRCUMFERENCE, HIP CIRCUMFERENCE, WAIST-HIP RATIO, AND PSORIASIS}

There was a graded association between waist circumference, hip circumference and the risk of incident psoriasis (Table 3). Compared with a waist circumference of 20-28 in (tertile 1), the multivariate RR of incident psoriasis for a waist circumference of 33-66 in (tertile 3) was 1.50 (95 CI\%, 1.24-1.82). In comparison to hip circumference of 20-37 in (tertile 1), the multivariate RR of incident psoriasis for a hip circumference of 41-79 in (tertile 3) was 1.44 (95 CI\%, 1.19-1.74). A higher waist-hip ratio was associated with an increased risk of incident psoriasis (multivariate RR, top vs bottom tertile, 1.40 (95\%CI, 1.15-1.70). There was no material difference in the multivariate RRs (adjusted for age, alcohol consumption, and smoking status) and RRs adjusted only for age. When simultaneously adjusted with BMI in addition to the multivariate models, the associations between waist circumference, hip circumference, and waist hip ratio and psoriasis were substantially attenuated and became non-significant. The risk estimates of updated BMI in these multivariate models were the same as those in Table 2 ( $R R$ for one unit increment, 1.04, $\mathrm{P}<0.0001$ ).

\section{WEIGHT CHANGE AND PSORIASIS}

Most women experienced weight gain from the age of 18 years, whereas 5\% of the women lost weight. There was a graded association between weight change since age 18 and the risk of psoriasis (Table 4). After adjustment for covariates and BMI at age 18, compared with women who maintained weight $(-5.0$ to $+4.9 \mathrm{lb})$, the RR of a weight gains of $35 \mathrm{lb}$ or greater from age 18 was 1.79 (95\% CI, 1.29-2.47) for incident psoriasis. The RR associated with $10 \mathrm{lbs}$ increment was 1.08 (95\% CI, 1.06-1.11; $P$ for $10 \mathrm{lbs}$ increment, <0.0001) (Table 
4). There was no material change in the trend in these multivariate RRs and age-adjusted RR.

\section{DISCUSSION}

We evaluated the association between obesity and psoriasis in a large prospective cohort of older women. The other prospective study, Nurses' Health Study II, also published by our group demonstrated a similar association among younger women [3]. We used multiple measures of obesity, including BMI, waist circumference, hip circumference, waist-hip ratio, and the change in adiposity as assessed by weight gain since the age of 18 years. The outcome measures in this report were self-reported, physician-diagnosed psoriasis.

We found that all measures of adiposity were significant risk factors for the development of psoriasis. Risk increased with elevated levels of adiposity measures, demonstrating a strong and consistent dose-response relationship. We found that BMI was strongly associated with psoriasis, and the associations of waist circumference, hip circumference and waist-hip ratio were substantially attenuated by additionally adjusting for BMI, probably because these measures are highly correlated. The BMI at age of 18 years was not associated with psoriasis, which suggests that current weight and weight gain is more relevant.

Published data suggests that losing weight may improve psoriasis [22,23], and several case studies have shown that weight loss from gastric bypass surgery results in remission of psoriasis $[24,25]$. A recent study showed that moderate weight loss (i.e. 5-10\% of the body weight) increases the therapeutic response to a low dose of cyclosporine in obese patients with moderate-to severe chronic plaque psoriasis further supporting the relevance of obesity to psoriasis [26].Weight loss may decrease the degree of obesity-induced inflammation by lowering the level of circulating inflammatory cytokines. [27]. which may be translated to a reduced risk and severity of psoriasis.

While the uncertainty remains about the exact association between obesity and psoriasis [10, 28], our prospective longitudinal data from incident case analysis further suggests that increased adiposity precedes the occurrence of psoriasis. As a marker of central obesity, higher waist-hip ratio was associated with an increasing risk of psoriasis. However, the association was attenuated after controlling for BMI, suggesting that central obesity is less relevant. One alternative explanation could be that there is more measurement error for waist and hip circumferences than the BMI. It would be important to reinforce the distinct possibility of psoriasis and obesity not being reciprocally or unidirectionally causal and rather deriving from a common underlying pathophysiology as obesity-associated inflammation has been shown to be mediated by leptin. It is an adipocytokine produced by adipose tissue that induces interleukin (IL)-6 and tumor necrosis factor (TNF)-a production and down-regulates interleukin (IL)-10, driving naive T cell polarization toward a Th1 phenotype which is the effector response in psoriasis [29].

There are several strengths and limitations of our study. To our knowledge, it is one of the largest, prospective assessments of multiple markers of adiposity in relation to the risk of psoriasis. The measures of body size used were self-reported; however, these measures have 
been validated and their accuracy has been demonstrated [19]. The onset of psoriasis was also self-reported, and the data have not been validated. Similar to other population based epidemiological studies of psoriasis; we did not clinically confirm the nurses' self-reported physician diagnosis of psoriasis with an examination by a dermatologist [30]. We expect the overall accuracy of self-reported physician diagnosis of psoriasis to be higher among registered nurses, as was the case with other health data in our cohort. As noted before, the validation rate of psoriasis in-the Nurses' Health Study II, a similar cohort, is 92\% for psoriasis (manuscript under submission).

The restriction to registered nurses in our cohort has its strengths and limitations. The cohort of well-educated women minimizes the potential for confounding associated with socioeconomic status, while providing high-quality data, with minimal loss to follow-up. Although the absolute rates of psoriasis and prevalence of obesity may not be representative of a random sample of US women, the biological effects of adiposity should be similar. Our findings would be most directly generalizable to white women with no history of psoriasis with the second peak of the reported bimodal peaks of psoriasis onset time (23 and 55 years).

In conclusion, this prospective study suggests that increased adiposity and weight gain are significant risk factors for psoriasis in women. Our findings provide further evidence for a potentially common inflammatory pathway linking obesity to psoriasis, emphasizing that psoriasis is indeed a systemic disease. With mounting evidence in this field, interventions to tackle obesity in the individual with psoriasis seem reasonable including lifestyle factors and dietary recommendations.

\section{Acknowledgments}

We are indebted to the participants in the Nurses' Health Study for their dedication and commitment.

Funding support: The work is supported by P01 CA87969.

Role of the Sponsors: The sponsors had no role in the design and conduct of the study; in the collection, analysis, and interpretation of data; or in the preparation, review, or approval of the manuscript.

\section{REFERENCES}

1. Gelfand JM, Stern RS, Nijsten T, et al. The prevalence of psoriasis in African Americans: results from a population-based study. J Am Acad Dermatol. 2005; 52:23. [PubMed: 15627076]

2. Gladman DD. Psoriatic arthritis. Dermatol Ther. 2004; 17:350-363. [PubMed: 15379770]

3. Setty AR, Curhan G, Choi HK. Smoking and the risk of psoriasis in women: Nurses' Health Study II. Am J Med. Nov; 2007 120(11):953-9. [PubMed: 17976422]

4. Qureshi AA, Dominguez PL, Choi HK, Han J, Curhan G. Alcohol Intake and Risk of Incident Psoriasis in US Women: A Prospective Study. Arch Dermatol. Aug 16.2010

5. Qureshi AA, Choi HK, Setty AR, Curhan GC. Psoriasis and the risk of diabetes and hypertension: a prospective study of US female nurses. Arch Dermatol. Apr; 2009 145(4):379-82. [PubMed: 19380659]

6. Sterry W, Strober BE, Menter A, on behalf of the International Psoriasis Council. Obesity in psoriasis: the metabolic, clinical and therapeutic implications. Report of an interdisciplinary conference and review.

7. WHO. 2000:6. 
8. Haslam DW, James WP. Obesity. Lancet. 2005; 366:1197-209. [PubMed: 16198769]

9. Shirai K. Obesity as the core of the metabolic syndrome and the management of coronary heart disease. Curr Med Res Opin. 2004; 20:295-304. [PubMed: 15025838]

10. Herron MD, Hinckley M, Hoffman MS, et al. Impact of obesity and smoking on psoriasis presentation and management. Arch Dermatol. 2005; 141:1527-1534. [PubMed: 16365253]

11. Naldi L, Chatenoud L, Linder D, et al. Cigarette smoking, body mass index, and stressful life events as risk factors for psoriasis: results from an Italian case-control study. J Invest Dermatol. 2005; 125:61-67. [PubMed: 15982303]

12. Huerta C, Rivero E, Rodriguez LA. Incidence and risk factors for psoriasis in the general population. Arch Dermatol. 2007; 143:1559-1565. [PubMed: 18087008]

13. Neimann AL, Shin DB, Wang X, et al. Prevalence of cardiovascular risk factors in patients with psoriasis. J Am Acad Dermatol. 2006; 55:829-835. [PubMed: 17052489]

14. Lindegard B. Diseases associated with psoriasis in a general population of 159,200 middle-aged, urban, native Swedes. Dermatologica. 1986; 172(6):298-304. [PubMed: 3089849]

15. Henseler T, Christophers E. Disease concomitance in psoriasis. J Am Acad Dermatol. 1995; 32:982-6. [PubMed: 7751469]

16. Mease P, Goffe B, Metz J, et al. Etanercept in the treatment of psoriatic arthritis and psoriasis: a randomized trial. Lancet. 2000; 356:385-90. [PubMed: 10972371]

17. Krueger G, Papp K, Stough D, et al. A randomized, double-blind, placebo-controlled phase III study evaluating efficacy and tolerability of 2 courses of alefacept in patients with chronic plaque psoriasis. J Am Acad Dermatol. 2002; 47:821-33. [PubMed: 12451365]

18. Setty AR, Curhan G, Choi HK. Obesity, waist circumference, weight change, and the risk of psoriasis in women: Nurses’ Health Study II. Arch Intern Med. 2007; 167:1670-1675. [PubMed: 17698691]

19. Rimm EB, Stampfer MJ, Colditz GA, Chute CG, Litin LB, Willett WC. Validity of self-reported waist and hip circumferences in men and women. Epidemiology. 1990; 1(6):466-473. [PubMed: 2090285]

20. Giovannucci E, Colditz G, Stampfer MJ, et al. The assessment of alcohol consumption by a simple self-administered questionnaire. Am J Epidemiol. 1991; 133(8):810-817. [PubMed: 2021148]

21. Hu FB, Willett WC, Li T, Stampfer MJ, Colditz GA, Manson JE. Adiposity as compared with physical activity in predicting mortality among women. N Engl J Med. 2004; 351:2694-2703. [PubMed: 15616204]

22. Higa-Sansone G, Szomstein S, Soto F, et al. Psoriasis remission after laparoscopic Roux-en-Y gastric bypass for morbid obesity. Obes Surg. 2004; 14:1132-4. [PubMed: 15479606]

23. Wolters M. Diet and psoriasis: experimental data and clinical evidence. Br J Dermatol. 2005; 153:706-14. [PubMed: 16181450]

24. Moll JM, Haslock I, Macrae IF, et al. Associations between ankylosing spondylitis, psoriatic arthritis, Reiter0s disease, the intestinal arthropathies, and Behcet0s syndrome. Medicine (Baltimore). 1974; 53:343-64. [PubMed: 4604133]

25. de Menezes Ettinger JE, Azaro E, de Souza CA, et al. Remission of psoriasis after open gastric bypass. Obes Surg. 2006; 16:94-7. [PubMed: 16417765]

26. Gisondi P, Del Giglio M, Di Francesco V, et al. Weight loss improves the response of obese patients with moderate-to-severe chronic plaque psoriasis to low-dose cyclosporine therapy: a randomized, controlled, investigator-blinded clinical trial. Am J Clin Nutr. 2008; 88:1242-7. [PubMed: 18996858]

27. Cancello R, Henegar C, Viguerie N, et al. Reduction of macrophage infiltration and chemoattractant gene expression changes in white adipose tissue of morbidly obese subjects after surgery-induced weight loss. Diabetes. 2005; 54(8):2277-2286. [PubMed: 16046292]

28. Lebwhol M, Callen JP. Obesity, smoking, and psoriasis. JAMA. 2006; 295(2):208-210. [PubMed: 16715548]

29. Ritchlin CT, Qureshi AA, de Vlam K, et al. Biomarkers in psoriasis and psoriatic arthritis: GRAPPA 2008. J Rheumatol. Feb; 2010 37(2):462-7. [PubMed: 20147482] 
30. Stern RS, Nijsten T, Feldman SR, Margolis DJ, Rolstad T. Psoriasis is common, carries a substantial burden even when not extensive, and is associated with widespread treatment dissatisfaction. J Investig Dermatol Symp Proc. 2004; 9(2):136-139. 


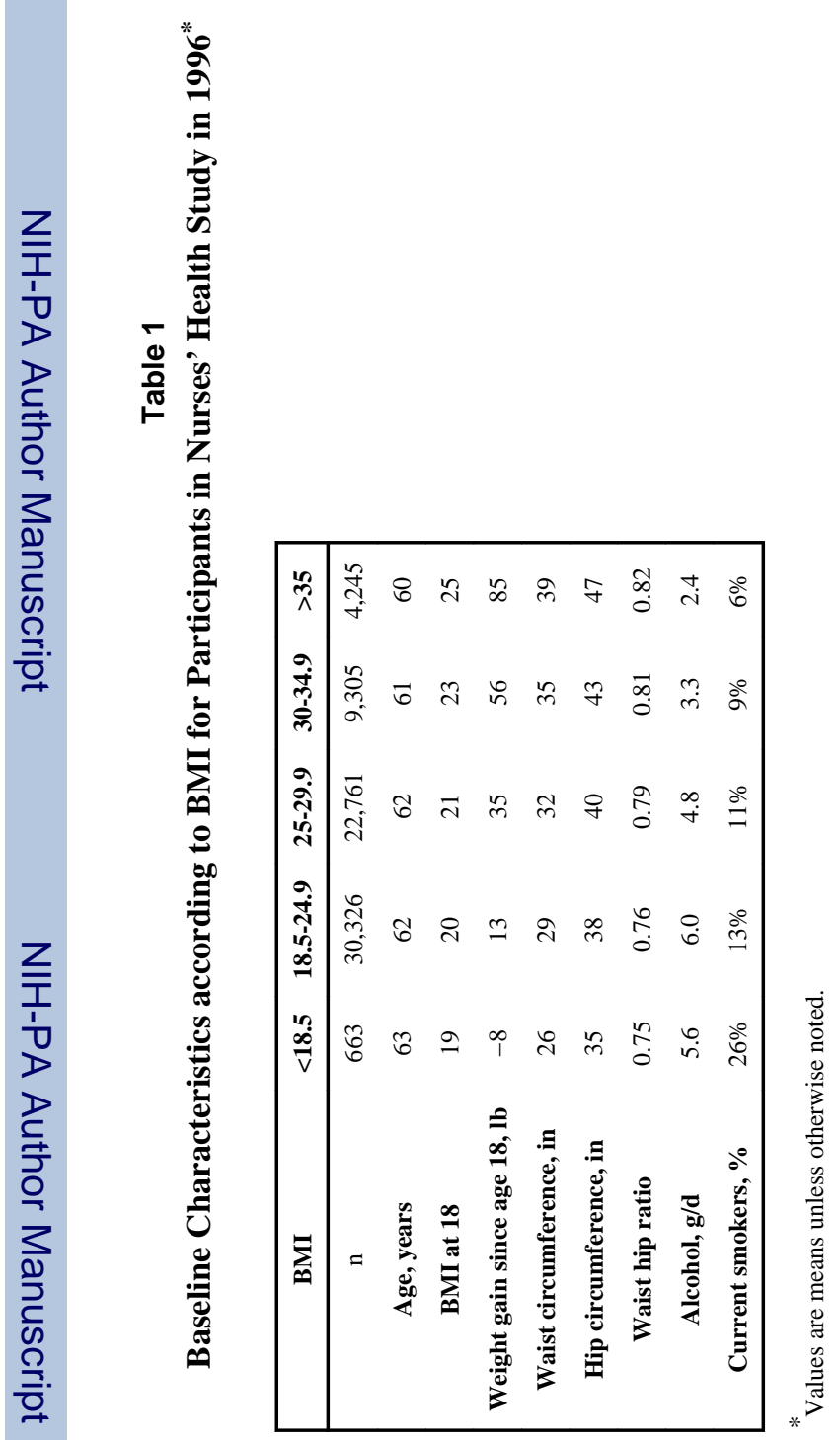

J Eur Acad Dermatol Venereol. Author manuscript; available in PMC 2014 September 30. 


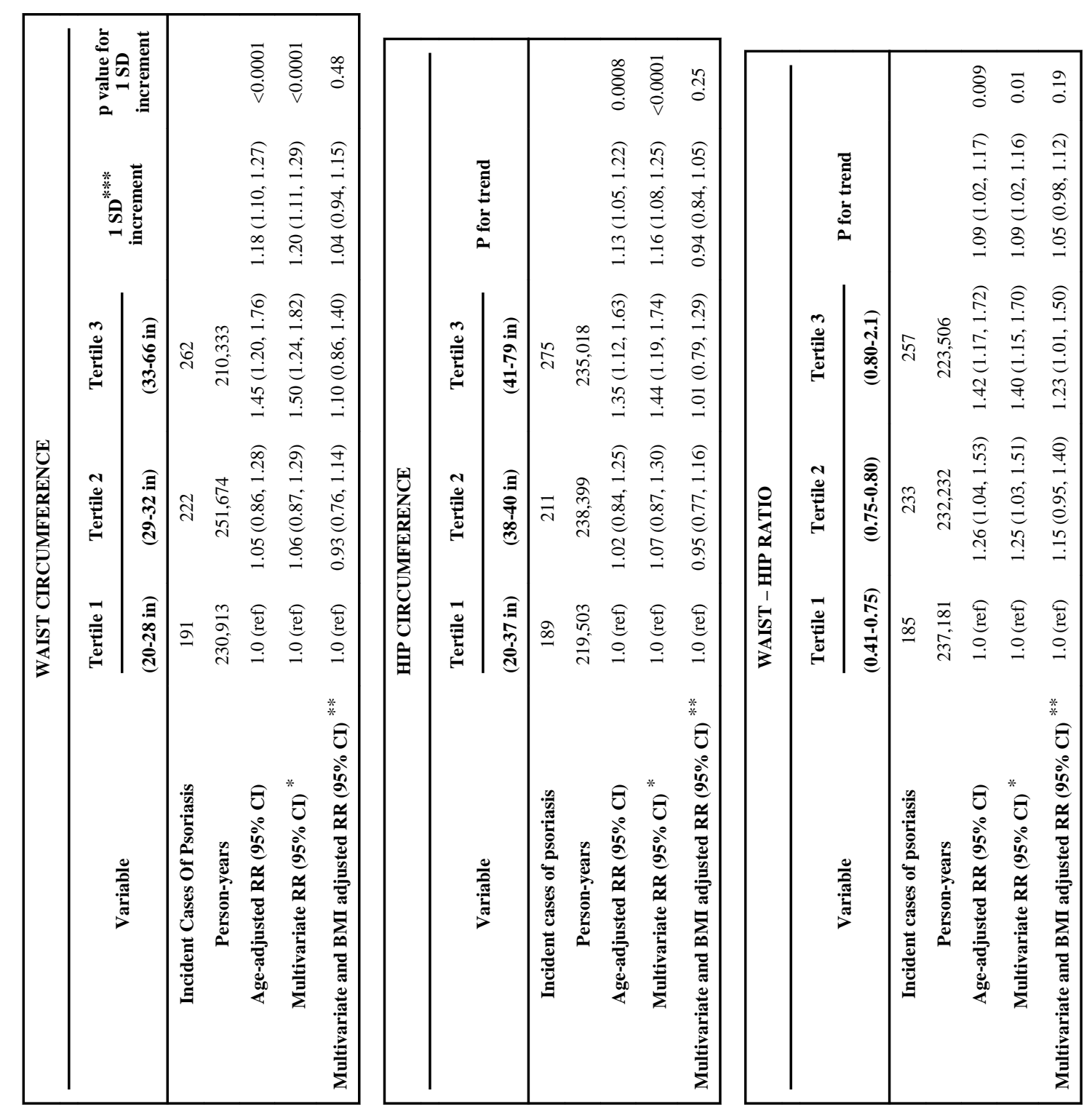

J Eur Acad Dermatol Venereol. Author manuscript; available in PMC 2014 September 30. 


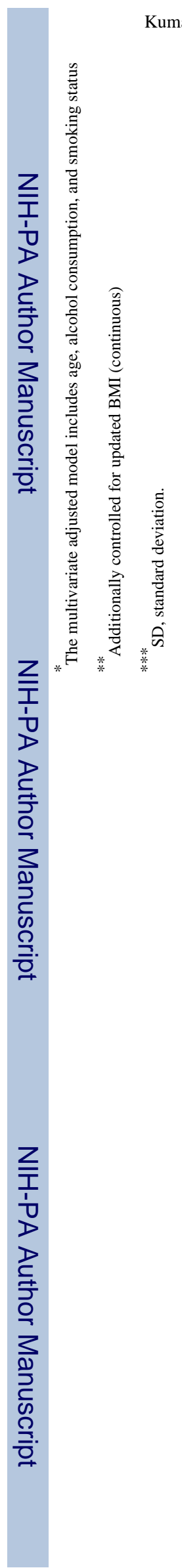

Kumar et al.

Page 13

J Eur Acad Dermatol Venereol. Author manuscript; available in PMC 2014 September 30. 


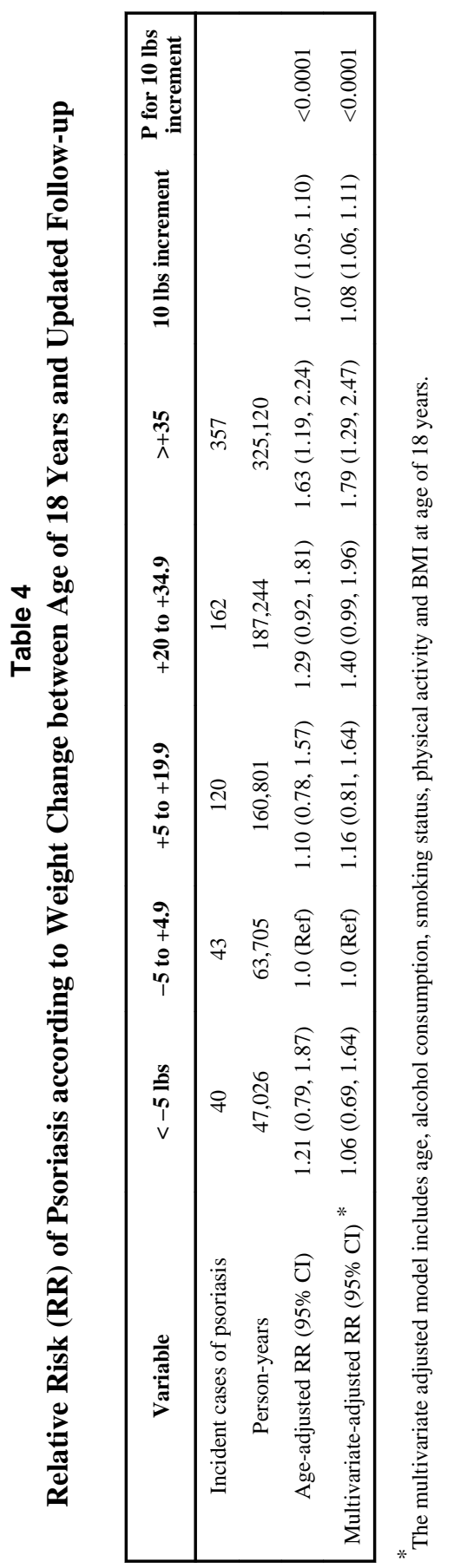

J Eur Acad Dermatol Venereol. Author manuscript; available in PMC 2014 September 30. 